\title{
Prevention of overweight and obesity in adult populations: a systematic review
}

\author{
Leslea Peirson PhD, James Douketis MD, Donna Ciliska PhD, Donna Fitzpatrick-Lewis MSc, \\ Muhammad Usman Ali MD MSc, Parminder Raina PhD
}

\section{Abstract}

Background: The prevalence of normal-weight adults is decreasing, and the proportion in excessive weight categories (body mass index $\geq 25$ ) is increasing. In this review, we sought to identify interventions to prevent weight gain in normal-weight adults.

Methods: We searched multiple databases from January 1980 to June 2013. We included randomized trials of primary care-relevant behavioural, complementary or alternative interventions for preventing weight gain in normal-weight adults that reported weight change at least 12 months after baseline. We included any studies reporting harms. We planned to extract and pool data for 4 weight outcomes, 6 secondary health outcomes and 5 adverse events categories.

Results: One small study provided moderate-quality evidence. The 12-month program, which used education and financial strategies and was offered more than 25 years ago in the United States, was successful in stabilizing weight and producing weight loss. More intervention participants maintained their baseline weight or lost weight than controls ( $82 \%$ v. $56 \%, p<0.0001)$, and program participants maintained their weight better than controls by showing greater weight reduction by the end of the intervention (mean difference adjusted for height $-0.82,95 \%$ confidence interval -1.57 to $-0.06, \mathrm{~kg}$ ). No evidence was available for sustained effects or for any other weight outcomes, secondary outcomes or harms.

Interpretation: We were unable to determine whether behavioural interventions led to weight-gain prevention and improved health outcomes in normal-weight adults. Given the importance of primary prevention, and the difficulty of losing weight and maintaining weight loss, this paucity of evidence is surprising and leaves clinicians and public health practitioners with unclear direction. Registration: PROSPERO no. CRD42012002753

verweight and obesity (body mass index [BMI] 25-29.9 and $\geq 30$, respectively), are global problems with increasing prevalence in most countries. ${ }^{1}$ Excess adiposity is related to a considerable increase in morbidity ${ }^{2-4}$ and premature mortality. ${ }^{5,6}$ The natural history of weight changes in adults has not been well studied, but data were collected on Canadian adults and analyzed for changes between 1996/97 and 2004/05. ${ }^{7}$ The overall change was average gain of $4 \mathrm{~kg}$ for men and $3.4 \mathrm{~kg}$ for women. ${ }^{7}$ Similarly, a large cohort study in the United States found that nonobese adults gain, on average, $0.8 \mathrm{lb}$ (about $0.36 \mathrm{~kg}$ ) annually. Another Canadian-based study showed that the prevalence of normalweight adults decreased by almost $7 \%$ between 2000/01 and 2011 , and the authors predicted a continued decline in this weight category, estimating that more than $55 \%$ of the adult population would be overweight or obese by $2019 .{ }^{9}$

Although a number of groups have produced clinical guidelines for overweight and obesity, there is an identified gap in knowledge regarding interventions that help maintain normal weight (BMI 18.5-24.9). ${ }^{10-15}$ Prevention is ideal, but it is not clear whether interventions for normal-weight people can prevent weight gain. We conducted a systematic review to address whether primary care-relevant interventions for normal- weight adults led to short-term or sustained weight-gain prevention or improved health outcomes.

\section{Methods}

The protocol was registered with PROSPERO (no. CRD42012002753) (www.crd.york.ac.uk/prospero).

\section{Search strategy}

We searched Embase, MEDLINE, Cochrane Central Register of Controlled Trials and PsycINFO from January 1980 to June 2013. The MEDLINE search strategy is provided as an example in Appendix 1 (available at www.cmajopen.ca /content/2/4/E268/suppl/DC1). References of primary studies included in this review and related systematic reviews were searched for studies not captured by our search.

Competing interests: See end of article.

This article has been peer reviewed.

Correspondence to: Leslea Peirson, peirson@mcmaster.ca

CMAJ Open 2014.DOI:10.9778/cmajo.20140019 


\section{PICOS statement}

The PICOS (population, intervention, comparator, outcome, setting) framework was as follows: (P) normal-weight adults aged 18 years or older; (I) behavioural, complementary or alternative interventions for weight-gain prevention; (C) no intervention, usual care or minimal component; $(\mathrm{O})$ change in weight, BMI, waist circumference or total body fat percentage, change in secondary health outcomes (lipids, glucose, blood pressure), and harms of interventions; and (S) generalizable to Canadian primary care settings. Additional details are provided in Box 1.

\section{Inclusion and exclusion criteria}

The inclusion and exclusion criteria are provided in Box 2.

\section{Study selection, quality assessment and data abstraction}

Titles and abstracts of papers were reviewed independently in duplicate. Any citation marked for inclusion by either team member went to full-text screening, which was also done independently in duplicate. Randomized trials were assessed using the Cochrane risk-of-bias tool. ${ }^{16}$ Overall, strength of the evidence (assessed as high, moderate, low or very low quality) was determined using the Grading of Recommendations

Box 1: Population, intervention, comparator, outcomes and setting

\section{Population}

- Normal-weight (BMI 18.5-24.9) adults aged $\geq 18 \mathrm{yr}$

\section{Interventions}

- Behavioural (diet, exercise and/or lifestyle), complementary or alternative (e.g., acupuncture, chiropractic, herbal supplements) interventions for preventing weight gain

\section{Comparator}

Intervention effectiveness

- No intervention, usual care or minimal intervention (e.g., newsletter or single information session on healthy living)

Intervention harms

- Any type of comparison group or no comparison group

Outcome

\section{Intervention effectiveness}

- Primary weight outcomes: change in weight $(\mathrm{kg}), \mathrm{BMI}$ and waist circumference; total body fat percentage; secondary health outcomes: change in total cholesterol, low-density lipoprotein cholesterol, fasting blood glucose, incidence of type 2 diabetes, and systolic and diastolic blood pressure

Intervention harms

- Labelling; disordered eating; psychological distress, such as anxiety, depression and stigma; nutritional deficits; cost burden

\section{Setting}

- Generalizable to Canadian primary care settings, or feasible for conducting in or referral from primary care; interventions should be initiated through (or feasible within) a primary care setting and (could be) delivered by a health care professional (e.g., physician, psychologist, nurse, dietician)

Note: $\mathrm{BMI}=$ body mass index.
Assessment, Development and Evaluation framework (GRADEpro version 3.2). One team member completed full data abstraction and a second verified all extractions. All data were re-verified before analyses. Interrater disagreements were resolved through discussion.

\section{Box 2: Inclusion and exclusion criteria}

Studies were included if they met the following criteria:

- Intervention involved a behavioural, complementary and/or alternative strategy for weight-gain prevention; behavioural interventions could include diet, exercise and/or lifestyle strategies (lifestyle strategies were typically referred to as such by study authors and often included counselling, education or support, and environmental changes in addition to diet and/or exercise); complementary and alternative interventions included strategies such as acupuncture, chiropractic treatment and herbal supplements

- Intervention targeted adults aged $\geq 18 \mathrm{yr}$ with normal weight (BMI 18.5-24.9)

- Population was unselected, selected for low cardiovascular disease risk, or selected for increased risk for cardiovascular disease, hypertension, dyslipidemia or type 2 diabetes; population could include some (but not all) people with cardiovascular disease

- Randomized controlled trial with no intervention, usual care or minimal component (e.g., single newsletter or information session on general health) comparison group (condition applied only to studies assessing intervention effectiveness)

- Sample included at least 30 participants per arm at both baseline and the minimum outcome assessment point

- Reported data for 1 or more specified weight outcomes (i.e., change in weight $[\mathrm{kg}]$, BMI, waist circumference, total body fat percentage)

- Reported data for outcomes of interest at least 12 mo after baseline assessment

- No restrictions on study design, comparison group, number of participants, weight outcome reporting or timing of assessment were applied to studies that reported data for harms

- Results were published in English or French

Studies were excluded for the following reasons:

- Intervention involved a faith-based approach, a pharmacologic strategy or a surgical procedure

- Intervention targeted people who were underweight (BMI $<18.5)$, or overweight or obese (BMI $\geq 25)$

- Population was limited to participants with cardiovascular disease, or specifically enrolled participants who were pregnant, had an eating disorder or a condition that predisposes weight gain (e.g., metabolic syndrome, polycystic ovary syndrome)

- Intervention was conducted in an in-patient hospital, institutional, school or occupational setting, or any setting deemed not generalizable to primary care, such as those with existing social networks among participants or the ability to offer intervention elements that could not be replicated in a primary health care setting; clinical institutions (hospital, metabolic units) were excluded because we believed these were unlikely to be primary prevention programs and would mostly include overweight or obese people

- Design was a case report, case series or chart review

- Only available results were published in a language other than English or French

Note: $\mathrm{BMI}=$ body mass index 


\section{Data analysis plan}

For meta-analyses, we planned to use posttreatment means and standard deviations (SDs) for continuous outcomes (e.g., weight in $\mathrm{kg}$ ) and number-of-events data for binary outcomes (e.g., incidence of type 2 diabetes). We intended to use the DerSimonian and Laird random-effects model with inverse variance method to generate summary measures of effect as mean difference for continuous outcomes and risk ratio for binary outcomes. ${ }^{17}$ For studies that did not report SDs, we planned to calculate this value from the reported standard error (SE) of the mean, or from the $95 \%$ confidence intervals $(\mathrm{CIs}) .^{18}$ For studies that provided neither SDs nor SEs for follow-up data, we would impute the SD from baseline values or included studies of similar sample size and for the same outcome. For studies with more than one intervention arm (e.g., 2 diet plus exercise arms, one community-based group and one correspondence course), we planned to pool the data to do a pair-wise comparison with the control group. Alternatively, if groups were substantively different (e.g., low-calorie diet, high-intensity aerobic exercise), we intended to include the data for each arm compared with the control group but split the sample size for the control group to avoid a unit-of-analysis error and double counting. ${ }^{16}$ Weight reported in pounds was to be converted to kilograms. Similarly, if total cholesterol, low-density lipoproteins and fasting glucose were reported in $\mathrm{mg} / \mathrm{dL}$, they would be converted to Canadian standard units (i.e., $\mathrm{mmol} / \mathrm{L}$ ). We planned to use the Cochran $\mathrm{Q}(\alpha=0.10)$ and $I^{2}$ statistic to quantify heterogeneity within and between subgroups. If sufficient data were available, sensitivity analyses would be performed to evaluate statistical stability and effect on statistical heterogeneity. Subanalyses were to be based on the following: type of intervention (diet, exercise, diet plus exercise, lifestyle), intervention duration ( $\leq 12 \mathrm{mo},>12 \mathrm{mo}$ ), sex, baseline cardiovascular disease risk status (high risk: identified risk factors and/or diagnosis of type 2 diabetes, hypertension, dyslipidemia; low risk; unselected population; or not specified) and the study's risk-of-bias rating (high, unclear, low).

\section{Results}

\section{Search}

The database search located 31974 unique studies. Manual searches and reviews of reference lists from recent (published 2012-2013) relevant systematic reviews located 15 additional studies. After full-text screening, only one study was located that met the inclusion criteria for this review (Figure 1).

\section{Included study}

We found one trial that met the inclusion criteria for a normalweight population. ${ }^{19}$ Twenty-five other studies were excluded because of samples of mixed-weight populations; this indirect evidence is reported in the adult overweight and obesity prevention review we prepared for the Canadian Task Force on Preventive Health Care. ${ }^{20}$ The included pilot study was a randomized controlled trial of a 12 -month education- and incentive-based intervention conducted in the US state of Minnesota in the 1980s. The study involved collaboration between researchers in the School of Public Health at the University of
Minnesota and staff at a local health department. A staged recruitment strategy began with mailed invitations to a random sample of 3000 adults out of about 6000 who attended a centre for cardiovascular risk factor screening in the previous 18 months and whose weight was recorded as normal (i.e., $<115 \%$ ideal weight as per 1983 Metropolitan Life Insurance Company tables). Because of study limitations, only $61 \%$ of those who indicated interest (422/690) were invited to attend an orientation session, and 219 people were enrolled (110 assigned to the intervention group; 109 assigned to the control group). Based on measures taken at the screening visit, the sample was $71 \%$ female, mean age was 45.9 years, mean BMI was 23.1 , mean systolic blood pressure was $114.1 \mathrm{~mm} \mathrm{Hg}$, mean diastolic blood pressure was $69.8 \mathrm{~mm} \mathrm{Hg}$, mean serum cholesterol level was $4.9 \mathrm{mmol} / \mathrm{L}$ and $8 \%$ of the participants smoked. The only significant baseline difference between intervention and control groups was the percentage of participants with prior involvement in formal weight-control programs (18\% intervention, $30 \%$ control).

To raise awareness, participants in the Pound of Prevention pilot program $(n=110)$ were mailed monthly newsletters containing information about weight-related issues (e.g., diet, exercise, psychology of weight management, association between weight and health). To encourage regular self-monitoring, on a monthly basis they were asked to mail a record of their weight and a description of any weight-control strategies they were using. To increase motivation, a financial incentive system was set up to withdraw $\$ 10$ per month from each intervention participant's personal bank account. The accumulated money was reimbursed with interest at any time on request or at the end of the 1-year study, on the condition that the participant's weight did not increase from baseline. Finally, about 6 months into the program a 4-session mini-course was offered to provide more

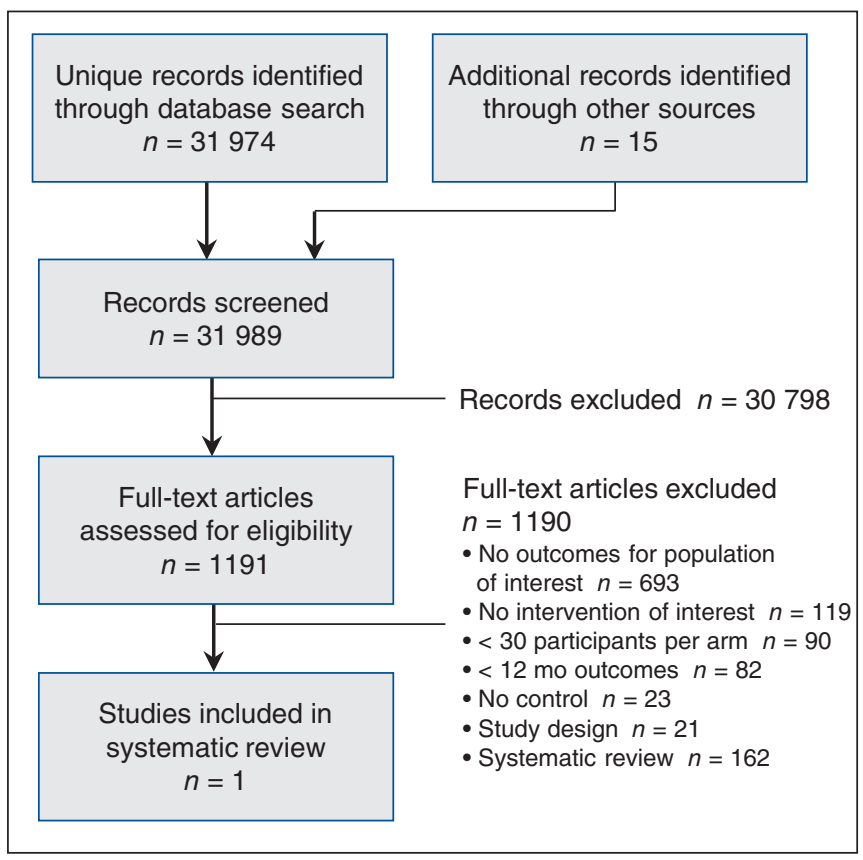

Figure 1: Selection of studies on interventions to prevent weight gain in normal-weight adults. 
extensive information and assistance on managing and losing weight through diet and exercise. Although open to all intervention participants, these sessions were primarily intended for those who experienced weight gain or who were unhappy about the amount of weight they gained during the first half of the program. Control participants $(n=109)$ had no contact with the program other than attending the orientation session when baseline measures were collected before group assignment and attending a follow-up visit for the 1-year outcome assessment.

\section{Outcomes}

There were insufficient trials to perform meta-analyses for any outcomes.

The included study ${ }^{19}$ reported only weight change in pounds (converted here to kilograms). All but 9 participants were included in the analysis. Five program participants dropped out because they moved and 2 discontinued their involvement for unspecified reasons. Two control participants were excluded from the analysis because medical conditions prevented taking weight measurements. More intervention participants $(n=103)$ maintained their baseline weight or lost weight during the 12 -month intervention than control participants $(n=108)(82 \%$ v. $56 \%, p<0.0001)$. Although both groups showed an overall reduction in weight from the baseline to postintervention assessments, our calculations show that program participants had a greater reduction in weight by the end of the intervention (mean difference adjusted for height $-0.82,95 \% \mathrm{CI}-1.57$ to $-0.06, \mathrm{~kg}$ ). No data were available to assess maintenance of weight-gain prevention, intervention effects on any other weight-related or secondary health outcomes, or adverse effects of program participation.

The available evidence was rated as moderate quality; downgrading occurred only for methodologic concerns identified through our assessment of the study's risk of bias. ${ }^{16}$ An overall unclear risk-of-bias rating was assigned because of the following: methods for generating the allocation sequence and concealing allocation were not described; it was not clear whether staff performing measures were unaware of group status; owing to the nature of behavioural interventions, participants and personnel were aware of group status; there was no mention of sample size calculations or power analyses; and those who expressed interest in participating may not have been representative of the general population (only $8 \%$ of those invited took part, and those who took part were more likely to be women, to not smoke and to have lower systolic blood pressure; they may have been more weight conscious than the general population and some may have been interested in losing weight).

\section{Interpretation}

We found only one trial of weight-gain prevention in normalweight participants. ${ }^{19}$ This is surprising considering the excess morbidity and mortality associated with overweight and obesity, the importance placed on prevention, the persistence of weight once gained and the difficulty in maintaining weight loss after any intervention. The paucity of evidence leaves clinicians and public health practitioners with unclear direction.
The included pilot study involving normal-weight participants reported moderate-quality evidence that educational strategies and financial incentives contributed to stabilized weight in the intervention group by the end of the 1-year program. Some adults in both groups lost weight, but fewer lost weight in the control group than in the intervention group. An increase in weight of less than $0.5 \mathrm{~kg}$ over one year may not appear clinically meaningful, but this should be considered with regard to weight gain that typically occurs in adults $(3-4 \mathrm{~kg} \text { in } 8 \mathrm{yr})^{7}$ and obesity-related health problems..$^{2-6}$ It is interesting that the later full trial of this intervention with more than 1000 participants (mean baseline BMI in overweight category) found no difference in weight gain at 3 years. ${ }^{21}$

This review was unable to address whether weight gain prevention is sustained or if preventing weight gain in normal-weight adults leads to other health improvements. Furthermore, no harms of interest to this review and no adverse effects of any kind were reported; therefore, we cannot comment on the likelihood of normal-weight adults experiencing adverse events as a result of participating in interventions for prevention of weight gain.

Data are still lacking regarding interventions that help normal-weight adults maintain their normal weight. A review of trials (26 studies) of mixed-weight populations provided indirect evidence that programs were successful in stabilizing weight and producing some weight loss by the end of the interventions. ${ }^{20}$ Intervention participants lost $0.73(95 \% \mathrm{CI}-0.93$ to -0.54$) \mathrm{kg}$ more, lowered their BMI by 0.24 (95\% CI -0.34 to -0.15$)$ more, reduced their waist circumference by an additional 0.95 (95\% CI -1.27 to -0.63$) \mathrm{cm}$ and lost $1.27 \%$ (95\% CI $-1.93 \%$ to $-0.61 \%)$ more total body fat than the control group. ${ }^{20}$ Similarly, a review of lifestyle interventions for preventing weight gain in young adults showed better weight maintenance among program participants than controls; intervention participants showed a modest mean decrease in body weight $(-0.87,95 \% \mathrm{CI}-1.56$ to -0.18 , $\mathrm{kg})$, whereas controls showed a modest mean increase $(0.86,95 \%$ CI 0.14 to $1.57, \mathrm{~kg}) .^{22}$ A slightly older review found 9 behaviourally based interventions that focused on weight-gain prevention among adults; 5 showed small but significant benefits in favour of the intervention groups whereas the remaining trials showed no effect. ${ }^{23}$ Another review with broader inclusion criteria found 11 trials and 11 observational studies that indicated there may be effective strategies to prevent weight gain, such as low-fat diets, eating fewer meals out of the home, consuming more fruits and vegetables, monitoring heart rate during exercise and participation in group lifestyle sessions with reminder text messages. ${ }^{24}$ Reviews that include studies with mixed-weight populations ${ }^{22-24}$ have critiques of the evidence that also apply to our findings: there is a small number of programs for weight-gain prevention, fewer still of high quality; substantial heterogeneity across strategies makes it difficult to comment on effective program features; the interventions and studies are typically of short duration so sustainability is unknown; and small samples means there may be insufficient power to detect change.

\section{Limitations}

This review found only one study reporting on the effectiveness of interventions to prevent weight gain in normal-weight adults. ${ }^{19}$ 
This single study was assessed as having unclear risk of bias, primarily due to the lack of information about sequence generation, allocation and blinding of outcome assessment, as well as concerns regarding study power and a high potential for selection bias. Also, results were available for only one specified weight outcome and none of the secondary outcomes or adverse effects of interest.

A language filter was applied in the original search because of limited resources available to appropriately handle papers in multiple languages (e.g., screening, translation and interpretation); including only publications in English or French meant papers about relevant interventions available only in other languages were not captured.

This review did not examine evidence for how long it takes a normal-weight person to become overweight or obese; this remains an unanswered question that may be the focus of future clinical research.

\section{Conclusion}

Because of very limited and dated evidence, we were unable to conclusively determine whether behaviourally based, primary care-relevant prevention programs lead to short-term or sustained weight-gain prevention and improved health outcomes in normal-weight adults. Despite increasing prevalence of overweight and obesity, and the enormity of the social, emotional, physical and economic sequelae, there are few upstream measures in place for true primary prevention (directed at the normal-weight population). Contemporary intervention research involving normal-weight samples with long-term follow-up is required to effectively answer this question.

In this review, people who were motivated to join a program for weight-gain prevention not only did not gain weight, but actually lost a small amount of weight. For adults with normal weight at baseline, this small weight loss may not be clinically meaningful, but it may offer protection against the health risks generally associated with weight gain. Interpreting these results is challenging, and it is difficult to know how normal-weight adults might be motivated to consider participating in such interventions.

\section{References}

1. Obesity and overweight. Geneva (Switzerland): World Health Organization; 2014. Available: www.who.int/mediacentre/factsheets/fs311/en (accessed 2014Jan. 28).

2. Eckersley RM. Losing the battle of the bulge: causes and consequences of increasing obesity. Med 7 Aust 2001;174:590-2.

3. Mokdad AH, Ford ES, Bowman BA, et al. Prevalence of obesity, diabetes, and obesity-related health risk factors, 2001. 7AMA 2003;289:76-9.

4. Wilson PW, D'Agostino RB, Sullivan L, et al. Overweight and obesity as determinants of cardiovascular risk: the Framingham experience. Arch Intern Med 2002;162:1867-72.

5. Flegal KM, Graubard BI, Williamson DF, et al. Excess deaths associated with underweight, overweight, and obesity. FAMA 2005;293:1861-7.

6. Allison DB, Fontaine KR, Manson JE, et al. Annual deaths attributable to obesity in the United States. FAMA 1999;282:1530-8

7. Orpana HM, Tremblay MS, Finès P. Trends in weight change among Canadian adults. Health Rep 2007;18:9-16.

8. Mozaffarian D, Hao T, Rimm EB, et al. Changes in diet and lifestyle and longterm weight gain in women and men. N Engl F Med 2011;364:2392-404.

9. Twells LK, Gregory DM, Reddigan J, et al. Current and predicted prevalence of obesity in Canada: a trend analysis. CMA7 Open 2014;2:E18-26.

10. Clinical practice guidelines for the management of overweight and obesity in adults. Canberra (Australia): National Health and Medical Research Council; 2013. Available: www.nhmrc.gov.au/_files_nhmrc/publications/attachments/n57 obesity_guidelines_130531.pdf(accessed 2014 Jan. 28).

11. Clinical guidelines for weight management in New Zealand adults. Wellington (New Zealand): Ministry of Health, Clinical Trials Research Unit; 2009. Available: www.health.govt.nz/system/files/documents/publications/weight-management -adults-guidelines.pdf (accessed 2014 Jan. 28).

12. Management of obesity: a national clinical guideline. Edinburgh (Scotland): Scottish Intercollegiate Guidelines Network (SIGN); 2010. Available: www.sign.ac.uk /pdf/sign 115.pdf (accessed 2014 Jan. 28).

13. Moyer VA; U.S. Preventive Services Task Force. Screening for and management of obesity in adults: U.S. Preventive Services Task Force recommendation statement. Ann Intern Med 2012;157:373-8.

14. Lau DC, Douketis JD, Morrison KM, et al. 2006 Canadian clinical practice guidelines on the management and prevention of obesity in adults and children [summary]. CMA7 2007;176:S1-13.

15. Obesity: guidance on the prevention, identification, assessment and management of overweight and obesity in adults and children [CG43]. London (UK): National Institute of Health and Clinical Excellence (NICE); 2006. Available: www.nice.org.uk /nicemedia/pdf/cg43niceguideline.pdf (accessed 2014 Jan. 28).

16. Cochrane handbook for systematic reviews of interventions. Version 5.1.0 ed. New York: John Wiley \& Sons; 2011.

17. DerSimonian R, Laird N. Meta-analysis in clinical trials. Control Clin Trials 1986;7:177-88.

18. Deeks, JJ, Higgins, JP, Altman, DG; Cochrane Methods Group. Analysing data and undertaking meta-analyses. In: Cochrane handbook for systematic reviews of interventions. Version 5.0.2 ed. Chichester (UK): John Wiley \& Sons, Ltd.; 2009. Chapter 9.

19. Forster JL, Jeffery RW, Schmid TL, et al. Preventing weight gain in adults: a pound of prevention. Health Psychol 1988;7:515-25.

20. Peirson, L, Fitzpatrick-Lewis, D, Ali, MU, et al. Prevention of overweight/obesity in adult populations: a systematic review with meta-analyses. Ottawa: Canadian Task Force on Preventive Health Care; 2014.

21. Jeffery RW, French SA. Preventing weight gain in adults: the pound of prevention study. Am 7 Public Health 1999;89:747-51.

22. Hebden L, Chey T, Allman-Farinelli M. Lifestyle intervention for preventing weight gain in young adults: a systematic review and meta-analysis of RCTs. Obes Rev 2012;13:692-710.

23. Lombard CB, Deeks AA, Teede HJ. A systematic review of interventions aimed at the prevention of weight gain in adults. Public Health Nutr 2009;12:2236-46.

24. Hutfless S, Gudzune KA, Maruthur N, et al. Strategies to prevent weight gain in adults: a systematic review. Am 7 Prev Med 2013;45:e41-51.

Affiliations: McMaster Evidence Review and Synthesis Centre (Peirson, Ciliska, Fitzpatrick-Lewis, Usman, Raina), Hamilton, Ont.; Department of Medicine (Douketis), McMaster University, Hamilton, Ont.; St. Joseph's Health Care Hamilton (Douketis), Hamilton, Ont.

Contributors: Leslea Peirson, Donna Ciliska, James Douketis, Donna Fitzpatrick-Lewis and Parminder Raina contributed to the conceptualization, data interpretation, writing and manuscript review. Leslea Peirson, Donna Ciliska and Donna Fitzpatrick-Lewis contributed to the methods, data collection and project coordination. Muhammad Usman Ali performed the data analysis and contributed to writing and manuscript review. All of the authors approved the version submitted for publication and agree to act as guarantors of the work.

Funding: The Canadian Institutes of Health Research provided funding for this review but had no role in the design, analyses, interpretation or decision to submit the paper for publication.

Acknowledgements: Parminder Raina holds a Tier 1 Canada Research Chair in Geroscience, and the Raymond and Margaret Labarge Chair in Research and Knowledge Application for Optimal Aging. Rachel Warren, Meghan Kenny, Eva Tsakonas and Mary Gauld contributed to the relevance and quality assessment, and data extraction phases. We are grateful to Maureen Rice for the literature search, and to Sharon Peck-Reid for database management and formatting of the report. The Public Health Agency of Canada Scientific Officers Sarah Connor Gorber and Amanda Shane contributed to the original protocol development and review of drafts of the technical report. Similarly, the Adult Obesity Working Group of the Canadian Task Force on Preventive Health Care members Paula Brauer, Maria Bacchus, Neil Bell, Elizabeth Shaw and Harminder Singh provided comments on the protocol and initial analyses.

Competing interests: James Douketis has participated in advisory boards for Bayer, Bristol-Myers-Squibb, Sanofi, AstraZeneca, Boehringer Ingelheim, Pfizer, Biotie, Portola and The Medicines Company; has consulted for Agen Biomedical, Ortho-Janssen Pharmaceuticals and Boehringer Ingelheim; has received grant support from Boehringer Ingelheim. No competing interests were declared by the other authors.

Supplemental information: For reviewer comments and the original submission of this manuscript, please see www.cmajopen.ca/content/2/4 /E268/suppl/DC1 